\title{
Effects of dexpanthenol on acetic acid-induced colitis in rats
}

\author{
YASIR FURKAN CAGIN ${ }^{1}$, HAKAN PARLAKPINAR $^{2}$, NIGAR VARDI $^{3}$, ALAADIN POLAT $^{4}$, \\ YAHYA ATAYAN $^{1}$, MEHMET ALI ERDOGAN ${ }^{1}$ and KEVSER TANBEK ${ }^{4}$ \\ Departments of ${ }^{1}$ Gastroenterology; ${ }^{2}$ Pharmacology; ${ }^{3}$ Histology and Embryology and \\ ${ }^{4}$ Physiology, Faculty of Medicine, Inonu University, Malatya 44280, Turkey \\ Received October 3, 2015; Accepted July 21, 2016
}

DOI: $10.3892 /$ etm.2016.3728

\begin{abstract}
While the pathogenesis of acetic acid (AA)-induced colitis is unclear, reactive oxygen species are considered to have a significant effect. The aim of the present study was to elucidate the therapeutic potential of dexpanthenol (Dxp) on the amelioration of colitis in rats. Group I ( $n=8$; control group) was intrarectally administered $1 \mathrm{ml}$ saline solution $(0.9 \%)$; group II [n=8; AA] was administered $4 \%$ AA into the colon via the rectum as a single dose for three consecutive days; group III ( $\mathrm{n}=8$; AA + Dxp) was administered AA at the same dosage as group II from day 4, and a single dose of Dxp was administered intraperitoneally; and group IV (n=8; Dxp) was administered Dxp similarly to Group III. Oxidative stress and colonic damage were assessed via biochemical and histologic examination methods. AA treatment led to an increase in oxidative parameters and a decrease in antioxidant systems. Histopathological examination showed that AA treatment caused tissue injury and increased caspase-3 activity in the distal colon and triggered apoptosis. Dxp treatment caused biochemical and histopathological improvements, indicating that Dxp may have an anti-oxidant effect in colitis; therefore, Dxp may be a potential therapeutic agent for the amelioration of IBD.
\end{abstract}

\section{Introduction}

Inflammatory bowel disease (IBD) describes a group of chronic diseases, including ulcerative colitis and Crohn's disease, which have a significant effect on life quality (1). While the pathogeneses of these diseases have not been fully identified, there is evidence to suggest that the immune system may have a causative role (2). Tissue injury occurs not only as a result of uncontrolled activation of the immune response, but also due to an increase in oxygen and nitrogen metabolites (3). Within this mechanism, reactive oxygen species (ROS) have

Correspondence to: Dr Yasir Furkan Cagin, Department of Gastroenterology, Faculty of Medicine, Inonu University, Elazig Road, Malatya 44280, Turkey

E-mail: yafur@hotmail.com

Key words: dexpanthenol, acetic acid, colon, oxidative stress been demonstrated to have a significant propagation effect on the pathogenesis of IBD, and has a dominant role in oxidative stress and apoptosis development (4). Oxidative stress, and the subsequent increase in apoptosis, have constituted the basis of the treatment strategy for the past 50 years; therefore, the majority of research into therapeutic agents in this field has aimed to eliminate $\operatorname{ROS}(5)$.

Acetic acid (AA)-induced colitis is one of several models of experimental colitis used to investigate IBD, and is propagated by intrarectal administration of AA to induce inflammation and ulceration in the rectum and the colon in rats. Oxidative destruction is thought to be the pathogenetic factor in this scenario, and this damage is also observed in the gross morphology of the colon (6). It is assumed that the same result occurs in colitis in humans (7).

Dexpanthenol (Dxp) is a biologically active alcohol-analog of pantothenic acid (PA) that is converted into PA inside the cell (8). PA exerts its anti-oxidative effects by increasing the synthesis of reduced glutathione (GSH) and associated peroxidase enzymes, which serve as the most important protective systems against oxidative stress and lipid peroxidation (9). Additionally, PA is incorporated into the structure of coenzyme A, stimulates epithelization, and exerts anti-inflammatory effects (10). The present study aimed to investigate the effects of Dxp on tissue damage and oxidative stress in a rat model of acetic acid-induced colitis, using biochemical and histopathological analyses. This will elucidate whether or not Dxp is a potential therapeutic agent for IBD.

\section{Materials and methods}

Animals. The present study was approved by the Institutional Ethics Committee for Animal Experiments (2014/A-41). All experiments were performed at the Inonu University Experimental Animals Production Center (IUEAPC; Malatya, Turkey). The study protocol was executed in accordance with the United States Animal Welfare Act and the Guide for the Care and Use of Laboratory Animals (National Institutes of Health, publication no. 5377-3, 1996), on the principles of animal research. A total of 32 Wistar albino female rats (3-4 months old; weight, $200 \pm 20 \mathrm{~g}$ ) were used. Rats were purchased from the IUEAPC and randomly divided into groups ( $n=8 /$ group). Each rat was kept in a separate cage. All rats were fed a standard rat pellet and tap water diet, with 12-h fasting employed prior to the experiment. Rats were 
maintained in an environment with a 12:12 light-dark cycle at $21 \pm 2^{\circ} \mathrm{C}$ room temperature and $60 \pm 5 \%$ humidity.

Induction of colitis. AA was used to induce acute colitis in rats. Rats were administered 4\% AA at 24-h intervals for three days with a $6 \mathrm{G}$ nelaton catheter, under mild anesthesia [ketamine hydrochloride $(50 \mathrm{mg} / \mathrm{kg})$ and xylazine $(5 \mathrm{mg}$ / $\mathrm{kg}$ ) mixture]. A catheter was inserted $6 \mathrm{~cm}$ into the anus, and $1 \mathrm{ml} \mathrm{AA}$ was administered followed by $1 \mathrm{ml}$ air, after which rats were maintained in the Trendelenburg position for $15 \mathrm{~min}$. Dxp was administered as a single dose of $500 \mathrm{mg} /$ $\mathrm{kg}$ for three days, under mild anesthesia (as described above). An insulin injector (Beybi Plastik Fabrikasi Sanayi A.Ş, Istanbul, Turkey) was used to deliver Dxp into the peritoneum from the right lower quadrant of the abdomen. Dosages of AA and Dxp were adjusted according to previous dose-response studies $(4,11)$.

Experimental protocol. A total of 32 rats were randomly divided into four equal groups $(n=8)$; i) group $I$, the control group, $1 \mathrm{ml}$ saline solution $(0.9 \%)$ was administered intrarectally; ii) group II, rats received $4 \%$ AA ( $1 \mathrm{ml} /$ day into the colon intrarectally) as a single dose for three consecutive days; iii) group III, rats received $4 \% \mathrm{AA}(1 \mathrm{ml} /$ day into the colon intrarectally) as a single dose for three consecutive days, and from day four, a single dose of Dxp $(500 \mathrm{mg} / \mathrm{kg}$; Bepanthene ampule $^{\circledR}$; Bayer AG, Leverkusen, Germany) was administered intraperitoneally (IP) for three days; and iv) group IV, $500 \mathrm{mg} / \mathrm{kg}$ Dxp was administered IP as a single dose for three consecutive days.

On day 7, laparotomy was performed under high-dose anesthesia (70 mg/kg ketamine and $8 \mathrm{mg} / \mathrm{kg}$ xylazine; i.m.), and tissue specimens were subsequently collected from the area $10-\mathrm{cm}$ distal from the colon. Oxidative stress markers, including malondialdehyde (MDA), total oxidant status (TOS), oxidative stress index (OSI), and anti-oxidant system markers, including superoxide dismutase (SOD), catalase (CAT), glutathione peroxidase (GPX), glutathione (GSH) and total anti-oxidant capacity (TAC) levels, were analyzed. Histopathological examination was performed under light microscopy.

Histological analyses. For light microscopic analysis, samples harvested from the distal colon were fixed in $10 \%$ formalin for $48 \mathrm{~h}$, dehydrated in an ascending alcohol series, and subsequently embedded in paraffin. Using a microtome, paraffin blocks were prepared for sectioning at $5-\mu \mathrm{m}$ thickness. Sections were stained with hematoxylin and eosin to assess the general morphology and Periodic acid-Schiff was employed for goblet cell secretion assessment.

Assessment of colonic injury was performed using the following criteria: Mucosal epithelium (ulceration); lamina propria (polymorphonuclear cell infiltrate); crypts loss, submucosa (hemorrhage, edema, infiltration of inflammatory cells); and caspase-3 immuno-reactivity (according to the extent of cell staining in all layers of the colon). Colonic mucosal damage was evaluated on a 0-4 scale by two histologists, who were blinded to the experimental group. Grading criteria were as follows: Grade 0, normal appearance; grade 1, slight injury (0-25\% involvement); grade 2 , moderate injury (25-50\% involvement); grade 3, severe injury (50-75\% involvement); and grade 4, extensive full thickness injury (>75\%) (12). The microscopic grade of each tissue was calculated as the sum of the scores given to each criteria; therefore, the maximum score that could be obtained was 28 . Surface goblet cells and mitosis figures in crypts were counted using a Leica Q Win image analysis system (Leica Microsystems Ltd., Milton Keynes, UK) in 20 areas under X40 objective.

For immunohistochemical analysis, sections of $4 \mu \mathrm{m}$ thickness were placed on polylysine-coated slides. Following rehydration, samples were immersed in a citrate buffer ( $\mathrm{pH} 7.6)$, heated in a microwave oven for $20 \mathrm{~min}$, cooled for $20 \mathrm{~min}$ at room temperature, and subsequently washed with phosphate-buffered saline (PBS; three 2 min washes). Washed sections were immersed in $0.3 \% \mathrm{H}_{2} \mathrm{O}_{2}$ for 7 min prior to washing again with PBS. Sections were incubated with a primary caspase-3 (CPP32) Ab-4 rabbit polyclonal antibody (cat no. RB-1197-R7; ready-to-use; Thermo Fisher Scientific, Inc.) for $30 \mathrm{~min}$ at room temperature, rinsed in PBS and subsequently incubated with biotinylated goat anti-polyvalent (cat. no. TP-125-BN; ready-to-use) antibody and streptavidin peroxidase (cat no. TS-125-HR) (both Thermo Fisher Scientific, Inc., Waltham, MA, USA) for $10 \mathrm{~min}$ at room temperature. Staining was completed with chromogen substrate for $15 \mathrm{~min}$ and slides were counter-stained with Mayer's hematoxylin for $1 \mathrm{~min}$, rinsed in tap water and dehydrated. Sections were examined by a histopathologist unaware of the experimental protocol using a Leica DFC 280 light microscope (Leica Microsystems, Ltd.). Caspase-3-positive cells in the cytoplasm were stained brown.

Biochemical analysis. A total of $200 \mathrm{mg}$ frozen colonic tissue was cut into sections using liquid nitrogen and homogenized in 10 volumes of ice-cold Tris- $\mathrm{HCl}$ buffer with respect to tissue weight (50 mmol/1; pH 7.4) using a homogenizer (Ultra Turrax IKAT18 basic homogenization; Werke, Staufen, Germany) for $3 \mathrm{~min}$ at 7,500 $\mathrm{x} g$ and $4^{\circ} \mathrm{C}$. The supernatant solution was extracted with an equal volume of ethanol/chloroform mixture $(3 / 5 ; \mathrm{v} / \mathrm{v})$. Following centrifugation at $3,000 \mathrm{x} g$ for $30 \mathrm{~min}$ at $4^{\circ} \mathrm{C}$, the upper layer was used to analyze total tissue protein levels.

Determination of MDA. MDA content in the homogenates was determined spectrophotometrically by measuring the presence of thiobarbituric acid reactive substances (TBARS) (13). A total of $3 \mathrm{ml} \mathrm{1 \%}$ phosphoric acid and $1 \mathrm{ml} 0.6 \%$ thiobarbituric acid solution were added to $0.5 \mathrm{ml}$ homogenate and pipetted into a tube. The mixture was heated in boiling water for $45 \mathrm{~min}$ and, following cooling, the colored part was supplemented with $4 \mathrm{ml} n$-butanol. Absorbance was measured using a spectrophotometer (UV-1601; Shimadzu Corporation, Kyoto, Japan) at 532 and $520 \mathrm{~nm}$. Amount of lipid peroxide was calculated as TBARS of lipid peroxidation. Results were expressed in $\mathrm{nmol} / \mathrm{g}$ tissue according to a prepared standard graph, prepared using the measurements of the standard solutions (1,1,3,3-tetramethoxypropane).

Determination of protein content. Protein content of the samples was determined by the method outlined by Lowry et al (14), using bovine serum albumin as a standard. 
Determination of SOD activity. Total SOD activity was determined according to the method used by Sun et al (15), which is based upon the principle of the inhibition of nitroblue-tetrazolium (NBT) reduction by the xanthine-xanthine oxidase system as a superoxide generator. One unit of SOD was defined as the enzyme amount required to induce a $50 \%$ reduction in the NBT reduction rate. SOD activity was calculated as $\mathrm{U} / \mathrm{mg}$ protein.

Determination of CAT activity. CAT activity was determined according to the method outlined by Aebi and Suter (16). The principle of the assay is based on the determination of the rate constant $\left(\mathrm{k}, ~^{-1}\right)$ or $\mathrm{H}_{2} \mathrm{O}_{2}$ decomposition rate at $240 \mathrm{~nm}$. Results are provided as $\mathrm{k} / \mathrm{g}$ protein.

Determination of GPX activity. GPX activity was measured according to the method used by Paglia and Valentine (17). Briefly, in a tube containing NADPH, GSH, sodiumazide and glutathione reductase, an enzymatic reaction was initiated by the addition of $\mathrm{H}_{2} \mathrm{O}_{2}$, and the change in absorbance at $340 \mathrm{~nm}$ was recorded using a spectrophotometer. Activity was expressed in $\mathrm{U} / \mathrm{g}$ protein.

Determination of GSH content. Concentration of GSH in the homogenate was measured spectrophotometrically according to Ellman's method (17). Briefly, aliquots of tissue homogenate were mixed with distilled water and $50 \%$ trichloroacetic acid in glass tubes and centrifuged at 2,000 x $g$ for $15 \mathrm{~min}$ at $4^{\circ} \mathrm{C}$. Supernatants were mixed with $0.4 \mathrm{~mol}$ Tris buffer $(\mathrm{pH} \mathrm{8.9)}$ and $0.01 \mathrm{~mol} 5,5$ '-dithiobis (2-nitrobenzoic acid) (DTNB) was added. Following agitation of the reaction mixture, absorbance was measured at $412 \mathrm{~nm}$ within $5 \mathrm{~min}$ of the addition of DTNB against a blank sample that contained no homogenate. Absorbance values were extrapolated from a glutathione standard curve and provided as $\mathrm{GSH}(\mu \mathrm{M} / \mathrm{g}$ tissue).

Determination of TAC. TAC levels were determined using a novel automated colorimetric measurement method developed by Erel (18). In this method, a hydroxyl radical is produced by the Fenton reaction, which reacts with the colorless substrate, $O$-dianisidine, to produce a dianisyl radical that is bright yellow-brown in color. Following the addition of the sample, the oxidative reactions initiated by the hydroxyl radicals present in the reaction mix are suppressed by the antioxidant components of the sample. In turn, this prevents the color change and thereby provides an effective measurement of the total antioxidant capacity of the sample. This assay has been demonstrated to have excellent precision values $(<3 \%)$. Results were presented as mmol Trolox equivalent/l.

Determination of TOS. TOS was determined using a novel automated measurement method, developed by Erel (18). Oxidants present in the sample oxidize the ferrous ion-O-dianisidine complex to a ferric ion. The oxidation reaction is enhanced by glycerol molecules, which are abundantly present in the reaction medium. The ferric ion forms a colored complex with xylenol orange in an acidic medium. Color intensity, which can be measured spectrophotometrically, is correlated to the total amount of oxidant molecules present in the sample. The assay was calibrated with $\mathrm{H}_{2} \mathrm{O}_{2}$ and the results were expressed in terms of $\mu \mathrm{mol}$ $\mathrm{H}_{2} \mathrm{O}_{2}$ equivalent/1.

Measurement of OSI. The TOS to TAC ratio was accepted as the OSI, which is an indicator of the degree of the oxidative stress (20). The OSI value was calculated using the following formula: OSI (arbitrary unit)=TOS/TAC. The OSI value of the distal colon samples was also calculated as OSI (arbitrary unit).

Statistical analysis. Data were expressed as median (min-max) values or mean \pm standard deviation depending upon the overall variable distribution. Normality was assessed using the Shapiro-Wilk test. Normally distributed data were analyzed by one-way analysis of variance, followed by Tukey's post-hoc tests. Non-normally distributed data were compared by Kruskal Wallis $\mathrm{H}$ test among the groups. When significant differences were determined, multiple comparisons were carried out using the Mann Whitney U test with Bonferroni correction. Statistical analyses were performed using SPSS version 22.0 for Windows (IBM SPSS, Armonk, NY, USA). P<0.05 was considered to indicate a statistically significant difference.

\section{Results}

Body weight. No animal mortality occurred during the experimental period. A decrease in the body weights was observed among the groups prior to and following the experiments (data not shown), however, this difference was not significant.

Biochemical assessment. As demonstrated in Table I, a significant difference was detected in the MDA levels between group AA and the control group $(\mathrm{P}<0.05)$, and between the MDA levels in Group AA and Group AA + Dxp $(\mathrm{P}<0.05)$. A significant decrease was detected in SOD, CAT and GPX levels in Group AA, compared with the control group $(\mathrm{P}<0.05)$. When Group AA + Dxp and Group AA were compared, significant improvements were observed in SOD, CAT, GPX, and GSH parameters in Group AA + Dxp $(\mathrm{P}<0.05)$. Significant differences in TOS and OSI levels were also detected between Group AA and the control group $(\mathrm{P}<0.05)$. Furthermore, there were marked differences in TOS, TAC, and OSI levels, and a significant difference in TOS levels between Group AA and Group AA + Dxp $(\mathrm{P}<0.05)$.

Histological results. Tissue sections of rats in the control and Dxp groups exhibited normal large bowel structures, and the colonic mucosa was observed as intact (Fig. 1A and B). Numerous goblet cells were identified on the surface epithelium and crypt in the control and Dxp groups (Fig. 2A and B). Notable histological damage occurred in the AA group (score, $12.5 \pm 2.3$ ). The lamina epithelialis, lamina propria, muscularis mucosae, and the submucosa of the colon layers could not be distinguished from each other due to extensive inflammatory cell infiltration and ulceration (Fig. 3A). Diffuse inflammatory cells in the mucosa were predominantly composed of neutrophils. In addition to severe goblet cell depletion, an absence of crypts was observed (Fig. 3B).

The microscopic score of the AA + Dxp group was significantly reduced compared to the AA group $(\mathrm{P}<0.01)$; however, Dxp 

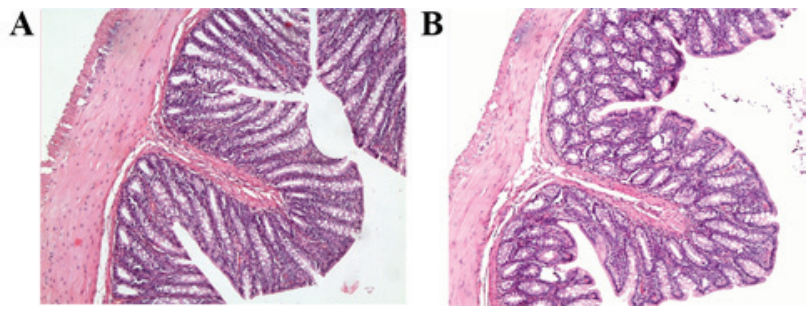

Figure 1. Regular epithelial line and intact crypt in the (A) control and (B) dexpanthenol groups, as detected by hematoxylin and eosin staining (magnification, $\mathrm{x} 33$ ).
A

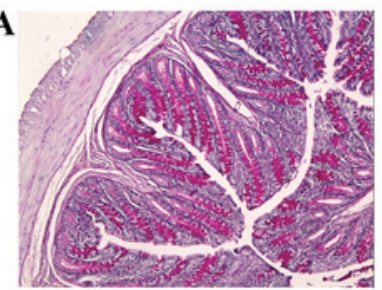

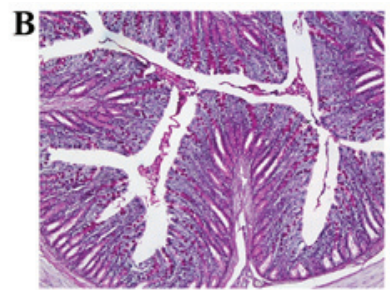

Figure 2. Abundant goblet cells in the surface and crypt epithelium, and Periodic acid-Schiff (+) reaction-induced magenta staining in the (A) control and (B) dexpanthenol groups (magnification, x33).
A

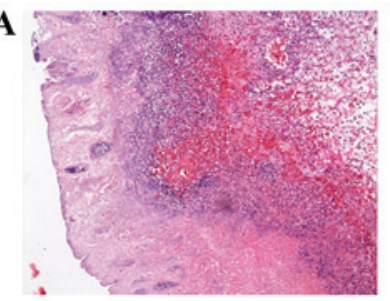

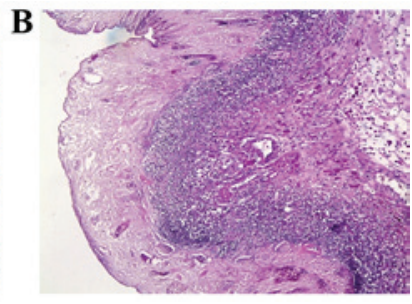

Figure 3. Acetic acid group. (A) Extensive epithelial loss, destruction of crypts and severe inflammatory cell infiltration, hemorrhage and sub-mucosal edema, as detected by hematoxylin and eosin staining. (B) Marked goblet cell depletion and absence of crypts, as detected by the Periodic acid-Schiff staining. Images were captured at x33 magnification.

treatment did not completely alleviate lesions, it merely restricted them. Ulceration, inflammatory cell infiltration and loss of crypt were still present in the affected areas (Fig. 4A). In some areas, the mucosa remained intact in this group. In intact fields, goblet cells were detected on the surface and crypt epithelium (Fig. 4B). Additionally, the number of mitotic figures was significantly increased when compared with the AA group $(\mathrm{P}<0.0001)$.

Caspase-3 immunostaining was only detected in the epithelial cells on the luminal surface in the control and Dxp groups (Fig. 5A and B). Conversely, caspase-3-positive cells were observed only in epithelial crypt cells due to superficial epithelial ulceration in the AA group (Fig. 5C). The appearance of cells stained with caspase- 3 in the AA + Dxp group was consistent with the control group (Fig. 5D). Results of histological grading and the number of goblet cells and mitotic figures in all groups are shown in Tables II and III.

\section{Discussion}

IBD describes a group of chronic diseases that have marked effects on the quality of life of those affected. In recent years, 
Table II. Colonic injury grades among the study groups.

\begin{tabular}{lcccc}
\hline Group & Control & AA & AA + Dxp & Dxp \\
\hline Microscopic grade & $1.1 \pm 0.1$ & $12.5 \pm 2.3^{\mathrm{a}}$ & $6.8 \pm 2.3^{\mathrm{b}}$ & $1.4 \pm 0.2$
\end{tabular}

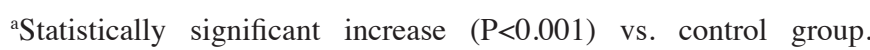

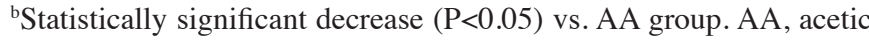
acid; Dxp, Dexpanthenol.

Table III. Number of goblet cells and mitotic figures.

\begin{tabular}{lcc}
\hline Group & Goblet cell & Mitotic figure \\
\hline Control & $62.0 \pm 17.3$ & $1.3 \pm 1.1$ \\
AA & $15.1 \pm 19.6^{\mathrm{a}}$ & $0.7 \pm 1.0^{\mathrm{a}}$ \\
AA + Dxp & $37.7 \pm 25.5^{\mathrm{b}}$ & $1.2 \pm 1.1^{\mathrm{b}}$ \\
Dxp & $61.8 \pm 17.2$ & $1.2 \pm 1.2$ \\
\hline
\end{tabular}

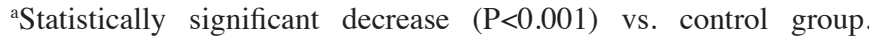

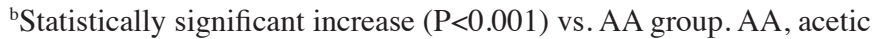
acid; Dxp, dexpanthenol.

the incidence of IBD has gradually increased (19). Despite the potential role of genetic, immunologic, and environmental factors, the etiology of IBD remains unclear; however, free oxygen radicals are considered to be a causal factor for IBD (20). Therefore, several studies have aimed to identify potential IBD therapies. A number of previous studies have focused on oxidative stress, which is important in mucosal injury pathogenesis (21) and is an initiator of apoptosis $(22,23)$. Apoptosis is important in tissue homeostasis (24); insufficient or excessive apoptosis disrupts equilibrium and causes various diseases (25). Apoptotic cell death also alters epithelial barrier function and allows infiltration by pathogenic microorganisms. While the details remain unclear, several studies (26-28) have suggested that apoptosis has a role in IBD pathogenesis, together with oxidative stress (29). Therefore, the majority of studies have focused on substances with anti-oxidant and anti-inflammatory properties.

In the present study, the oxidative stress, apoptosis and anti-oxidant properties of Dxp were investigated in a rat model of AA-induced colitis. The AA-induced colitis model used, is beneficial for the investigation of IBD pathogenesis and novel treatment options for ulcerative colitis, since it induces inflammation and ulceration (7).

Colon tissue injury was detected in the colitis model with a significant increase in oxidative stress markers and histopathological changes. Oxidative stress is considered to be a causative factor of AA-associated alterations in the colon tissue. Experimental studies have indicated that oxidative stress results from the shift of equilibrium between the pro-oxidant and anti-oxidant systems in favor of the pro-oxidant system as a result of excessive production of free oxygen radicals (30).

In the present study, distal colitis was induced via AA treatment, which revealed that AA treatment caused prominent tissue injury. When the results of the colonic tissue in the
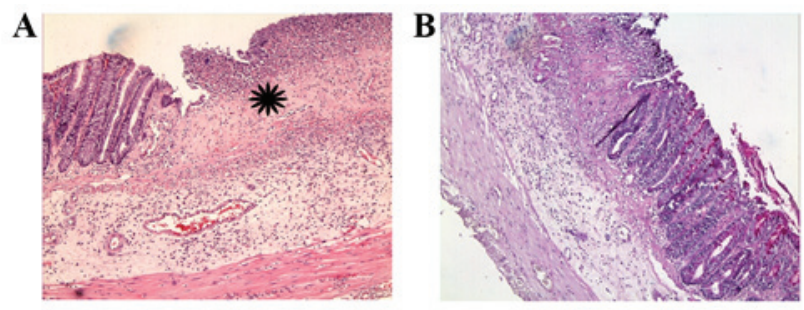

Figure 4. Acetic acid + dexpanthenol group. (A) Some mucosal areas were damaged (star), whereas other mucosal areas are intact. Degenerative changes and loss of crypts were detected in the injured field, following hematoxylin and eosin staining. (B) Goblet cells are visible, except in the affected area, following Periodic acid-Schiff staining. Images were captured at x33 magnification.
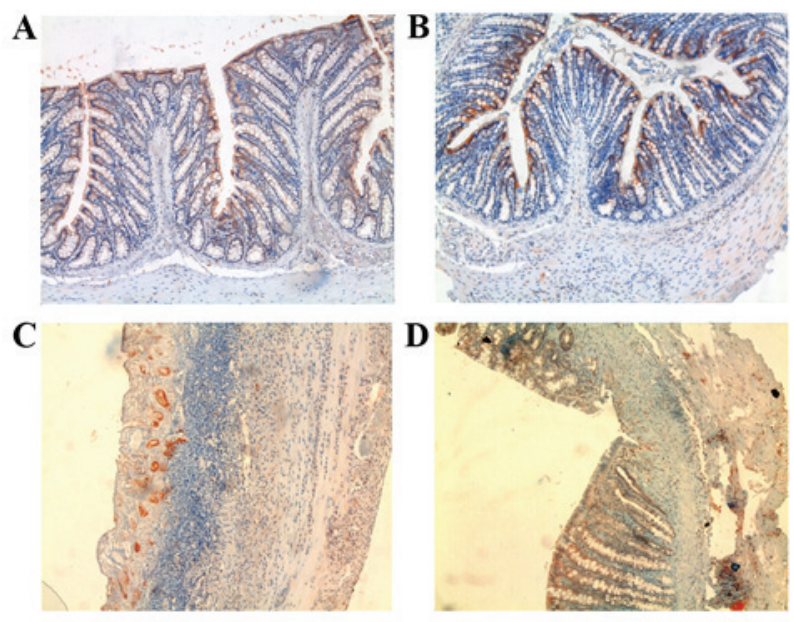

Figure 5. Diffuse caspase-3 staining in the surface epithelium of the (A) control and (B) dexpanthenol groups. (C) In the acetic acid-treated group, cells stained with caspase-3 were observed in the crypts. (D) Caspase-3-positive cells were only visible in the surface epithelium of unaffected areas. Images were captured at $\mathrm{x} 33$ magnification.

control group and Group AA were compared, AA treatment was demonstrated to induce an increase in MDA levels in the tissue. Therefore, it was determined that AA may have caused oxidative stress by leading to a marked increase in MDA levels in the colon tissue. MDA is the toxic end-product of lipid peroxidation, and reflects the level of lipid peroxidation in the tissue; hence its common usage as a marker of lipid peroxidation (31). MDA is secreted due to the toxic effects of active free oxygen radicals. While ROS affect all biomolecules in the organic environment, their major targets are membrane lipids, other lipids, proteins and DNA (32). ROS initiate lipid peroxidation by removing a hydrogen atom (33). Previous studies have shown that MDA levels are elevated in IBD (34-36). Furthermore increased lipid peroxidation has been reported in AA-associated tissue injuries, which is consistent with the findings of the present study. The present study demonstrated that Dxp treatment caused a significant decrease in AA-associated oxidative stress.

Another important finding of the present study was that AA treatment leads to a decrease in SOD, CAT and GPX levels in the tissue. It is well-known that enzymatic and non-enzymatic anti-oxidant systems exist to protect tissues from pro-oxidants and, as there is a balance between these systems, the imbal- 
ance has a role in the development of various diseases (37). SOD, CAT and GPX are endogenous enzymatic anti-oxidants, whereas GSH is a non-enzymatic anti-oxidant (38). These molecules protect cells and organisms from cytotoxic free oxygen radicals. SOD is among the most important enzymatic anti-oxidants. Oxygen radicals are converted into $\mathrm{H}_{2} \mathrm{O}_{2}$ by SOD. $\mathrm{H}_{2} \mathrm{O}_{2}$ is subsequently detoxified by CAT and GPX, and is converted into $\mathrm{H}_{2} \mathrm{O}$ and $\mathrm{O}_{2}$ molecules. The release of ROS in IBD disrupts the anti-oxidant system during mucosal inflammation and leads to oxidative damage. Decreased anti-oxidant levels have been reported in patients with ulcerative colitis $(39,40)$. Kruidenier et al (41) demonstrated that $\mathrm{Cu} / \mathrm{Zn}$ SOD and MnSOD levels were elevated in patients with IBD, compared with the control group. Kuralay et al (35) established an experimental model of colitis and indicated that SOD levels increased as a response to oxidative stress, and this increase was successfully reduced after treatment with anti-oxidant agents. The findings of the present study are consistent with the existing hypothesis of the mechanism of colitis tissue injury, which occurs as a result of ROS that weaken the anti-oxidant system. Furthermore, the results of the present study are consistent with previous studies, which have demonstrated increased lipid peroxidation and decreased anti-oxidant systems in AA-induced colitis (42).

The GSH cycle is the other important intracellular anti-oxidant defense system. It is used as a substrate for the activity of several anti-oxidant enzymes. In particular, GPX is a glutathione-dependent enzyme. In the presence of GSH, GPX detoxifies $\mathrm{H}_{2} \mathrm{O}_{2}$ into $\mathrm{H}_{2} \mathrm{O}$ and $\mathrm{O}_{2}$ molecules. GSH subsequently loses a hydrogen atom and oxidized glutathione (GSSG) is formed (43). Glutathione reductase forms GSH from GSSG. A decrease in GSH activity increases oxidative stress and leads to the accumulation of toxic products (44). In the present study, AA led to a decrease in GSH levels.

In the present study, increased levels of TOS and OSI, and decreased TAC levels were detected in Group AA. TOS and OSI levels in Group AA + Dxp were reduced compared with Group AA; however, no significant increase in TAC levels was detected. These findings are consistent with the results of a previous study (45), suggesting that AA increases oxidative stress, whereas Dxp decreases it.

Macroscopic and histopathological examinations are the gold standards for evaluating inflammatory injury in the colon. The present study demonstrated that caspase- 3 activity in the colon was considerably increased in Group AA compared with the control group. When Group AA + Dxp and Group AA were compared, Dxp treatment significantly decreased caspase-3 activity. This effect can be attributed to the anti-inflammatory and anti-apoptotic effects of Dxp. Altintas et al (39) have previously demonstrated that Dxp treatment significantly decreases tissue injury and apoptosis. The findings of the present study are consistent with this study. Despite these effects of Dxp, it is still unclear how Dxp exerts these effects at the molecular level.

The present study indicated that when Dxp was IP administered to rats with AA-induced colitis can reduce the extent of colonic mucosal damage, abate the increase in MDA, TOS and OSI levels, and restore diminished antioxidant enzymes and substances such as SOD, CAT, GPX, GSH and TAC. These effects can be attributed to the anti-oxidant, anti-inflammatory and anti-apoptotic effects of Dxp. These data also suggest that Dxp is more effective when administered after the induction of colitis.

In conclusion, the present study employed biochemical and histopathological analysis to demonstrate that oxidative stress and apoptosis are elevated in IBD. This, in turn, showed that oxidative stress and apoptosis may have a major role in IBD pathogenesis. These findings indicate that studies on IBD treatment will continue to aim to reduce the effects of the ROS. The beneficial effects of Dxp on tissue lipid peroxidation, protein oxidation, and anti-oxidant systems suggested that it may represent a treatment option to alleviate the spread of IBD.

\section{References}

1. Khor B, Gardet A and Xavier RJ: Genetics and pathogenesis of inflammatory bowel disease. Nature 474: 307-317, 2011.

2. Karlinger K, Györke T, Makö E, Mester A and Tarján Z: The epidemiology and the pathogenesis of inflammatory bowel disease. Eur J Radiol 35: 154-167, 2000.

3. Pavlick KP, Laroux FS, Fuseler J, Wolf RE, Gray L, Hoffman J and Grisham MB: Role of reactive metabolites of oxygen and nitrogen in inflammatory bowel disease. Free Radic Biol Med 33: 311-322, 2002.

4. Harputluoglu M, Demirel U, Yücel N, Karadăg N, Temel I, Firat S, Ara C, Aladağ M, Karincaoğlu M and Hilmioğlu F: The effects of Gingko biloba extract on acetic acid-induced colitis in rats. Turk J Gastroenterol 17: 177-182, 2006.

5. Zhu $\mathrm{H}$ and Li YR: Oxidative stress and redox signaling mechanisms of inflammatory bowel disease: Updated experimental and clinical evidence. Exp Biol Med (Maywood) 237: 474-480, 2012.

6. Pereira C, Grácio D, Teixeira JP and Magro F: Oxidative stress and DNA damage: Implications in inflammatory bowel disease. Inflamm Bowel Dis 21: 2403-2417, 2015.

7. Vishwakarma N, Ganeshpurkar A, Pandey V, Dubey N and Bansal D: Mesalazine-probiotics beads for acetic acid experimental colitis: Formulation and characterization of a promising new therapeutic strategy for ulcerative colitis. Drug Deliv 22: 94-99, 2015.

8. Slyshenkov VS, Rakowska M, Moiseenok AG and Wojtczak L: Pantothenic acid and its derivatives protect Ehrlich ascites tumor cells against lipid peroxidation. Free Radic Biol Med 19: 767-772, 1995.

9. Wojtczak L and Slyshenkov VS: Protection by pantothenic acid against apoptosis and cell damage by oxygen free radicals-the role of glutathione. Biofactors 17: 61-73, 2003.

10. Jessop CE and Bulleid NJ: Glutathione directly reduces an oxidoreductase in the endoplasmic reticulum of mammalian cells. J Biol Chem 279: 55341-55347, 2004.

11. Ceylan H, Yapici S, Tutar E, Ceylan NO, Tarakçığlu M and Demiryurek AT: Protective effects of dexpanthenol and y-27632 on stricture formation in a rat model of caustic esophageal injury. J Surg Res 171: 517-523, 2011.

12. Arribas B, Suárez-Pereira E, Ortiz Mellet C, García Fernández JM, Buttersack C, Rodríguez-Cabezas ME, Garrido-Mesa N, Bailon E, Guerra-Hernández E, Zarzuelo A and Gálvez J: Di-D-fructose dianhydride-enriched caramels: Effect on colon microbiota, inflammation, and tissue damage in trinitrobenzenesulfonic acid-induced colitic rats. J Agric Food Chem 58: 6476-6484, 2010.

13. Mihara M and Uchiyama M: Determination of malonaldehyde precursor in tissues by thiobarbituric acid test. Anal Biochem 86: 271-278, 1978.

14. Lowry OH, Rosebrough NJ, Farr AL and Randall RJ: Protein measurement with the Folin phenol reagent. J biol Chem 193: 265-275, 1951.

15. Sun Y, Oberley LW and Li Y: A simple method for clinical assay of superoxide dismutase. Clin Chem 34: 497-500, 1988.

16. Aebi $\mathrm{H}$ and Suter H: Catalase. In: Methods of Enzymatic Analysis. Bergmeyer HU (ed). Academic Press, New York, NY: p325, 1969.

17. Paglia DE and Valentine WN: Studies on the quantitative and qualitative characterization of erythrocyte glutathione peroxidase. J Lab Clin Med 70: 158-169, 1967. 
18. Erel O: A novel automated method to measure total antioxidant response against potent free radical reactions. Clin Biochem 37: 112-119, 2004

19. Burisch J, Jess T, Martinato M and Lakatos PL; ECCO-EpiCom: The burden of inflammatory bowel disease in Europe. J Crohns Colitis 7: 322-337, 2013

20. Martinez CA, Ribeiro ML, Gambero A, Miranda DD, Pereira JA and Nadal SR: The importance of oxygen free radicals in the etiopathogenesis of diversion colitis in rats. Acta Cir Bras 25: 387-395, 2010.

21. Kim YJ, Kim EH and Hahm KB: Oxidative stress in inflammation-based gastrointestinal tract diseases: Challenges and opportunities. J Gastroenterol Hepatol 27: 1004-1010, 2012

22. Thompson CB: Apoptosis in the pathogenesis and treatment of disease. Science 267: 1456-1462, 1995.

23. Franco R, Sánchez-Olea R, Reyes-Reyes EM and Panayiotidis MI: Environmental toxicity, oxidative stress and apoptosis: Ménage à trois. Mutat Res 674: 3-22, 2009.

24. Souza HS, Tortori CJ, Castelo-Branco MT, Carvalho AT, Margallo VS, Delgado CF, Dines I and Elia CC: Apoptosis in the intestinal mucosa of patients with inflammatory bowel disease: Evidence of altered expression of FasL and perforin cytotoxic pathways. Int J Colorectal Dis 20: 277-286, 2005.

25. Myers BS, Martin JS, Dempsey DT, Parkman HP, Thomas RM and Ryan JP: Acute experimental colitis decreases colonic circular smooth muscle contractility in rats. Am J Physiol 273: G928-G936, 1997.

26. Pedersen J, LaCasse EC, Seidelin JB, Coskun M and Nielsen OH: Inhibitors of apoptosis (IAPs) regulate intestinal immunity and inflammatory bowel disease (IBD) inflammation. Trends Mol Med 20: 652-665, 2014.

27. Bhattacharyya A, Chattopadhyay R, Mitra S and Crowe SE: Oxidative stress: An essential factor in the pathogenesis of gastrointestinal mucosal diseases. Physiol Rev 94: 329-354, 2014.

28. Becker C, Watson AJ and Neurath MF: Complex roles of caspases in the pathogenesis of inflammatory bowel disease. Gastroenterology 144: 283-293, 2013.

29. Weimann BI and Hermann D: Studies on wound healing: Effects of calcium D-pantothenate on the migration, proliferation and protein synthesis of human dermal fibroblasts in culture. Int $J$ Vitam Nutr Res 69: 113-119, 1999.

30. Halliwell B and Chirico S: Lipid peroxidation: Its mechanism, measurement, and significance. Am J Clin Nutr 57 (5 Suppl): 715S-724S; 725S, 1993

31. Kaya E, Gür ES, Ozgüç H, Bayer A and Tokyay R: L-glutamine enemas attenuate mucosal injury in experimental colitis. Dis Colon Rectum 42: 1209-1215, 1999.
32. Southorn P and Pow1s G: Free radicals in medicine. I. Chemical nature and biologic reactions. Mayo Clin Proc 63: 381-389, 1988

33. Cetinkaya A, BulbulogluE, Kurutas EB, Ciralik H, Kantarceken B and Buyukbese MA: Beneficial effects of $\mathrm{N}$-acetylcysteine on acetic acid-induced colitis in rats. Tohoku J Exp Med 206: 131-139, 2005.

34. Alzoghaibi MA, Al Mofleh IA and Al-Jebreen AM: Lipid peroxides in patients with inflammatory bowel disease. Saudi J Gastroenterol 13: 187-190, 2007.

35. Byrav D S P, Medhi B, Prakash A, Chakrabarti A, Vaiphei K and Khanduja KL: Comparative evaluation of different doses of PPAR- $\gamma$ agonist alone and in combination with sulfasalazine in experimentally induced inflammatory bowel disease in rats. Pharmacol Rep 65: 951-959, 2013.

36. Gul M, Kayhan B, Elbe H, Dogan Z and Otlu A: Histological and biochemical effects of dexmedetomidine on liver during an inflammatory bowel disease. Ultrastruct Pathol 39: 6-12, 2015.

37. Thomas MJ: The role of free radicals and antioxidants: How do we know that they are working? Crit Rev Food Sci Nutr 35: 21-39, 1995.

38. Flora G, Gupta D and Tiwari A: Toxicity of lead: A review with recent updates. Interdiscip Toxicol 5: 47-58, 2012.

39. Krzystek-Korpacka M, Neubauer K, Berdowska I, Zielinski B, Paradowski L and Gamian A: Impaired erythrocyte antioxidant defense in active inflammatory bowel disease: Impact of anemia and treatment. Inflamm Bowel Dis 16: 1467-1475, 2010.

40. D'Odorico A, Bortolan S, Cardin R, D'Inca' R, Martines D, Ferronato A and Sturniolo GC: Reduced plasma antioxidant concentrations and increased oxidative DNA damage in inflammatory bowel disease. Scand J Gastroenterol 36: 1289-1294, 2001.

41. Kruidenier L, Kuiper I, van Duijn W, Marklund SL, van Hogezand RA, Lamers CB and Verspaget HW: Differential mucosal expression of three superoxide dismutase isoforms in inflammatory bowel disease. J Pathol 201: 7-16, 2003.

42. Nieto N, Torres MI,Fernández MI, Girón MD, Ríos A, Suárez MD and Gil A: Experimental ulcerative colitis impairs antioxidant defense system in rat intestine. Dig Dis Sci 45: 1820-1827, 2000.

43. El-Beltagi HS and Mohamed HI: Reactive oxygen species, lipid peroxidation and antioxidative defense mechanism. Notulae Botanicae Horti Agrobotanici Cluj-Napoca 41: 44-57, 2013.

44. Hagar HH, El-Medany A, El-Eter E and Arafa M: Ameliorative effect of pyrrolidinedithiocarbamate on acetic acid induced colitis in rats. Eur J Pharmacol 554: 69-77, 2007.

45. Ermis H, Parlakpinar H, Gulbas G, Vardi N, Polat A, Cetin A, Kilic T and Aytemur ZA: Protective effect of dexpanthenol on bleomycin-induced pulmonary fibrosis in rats. Naunyn Schmiedebergs Arch Pharmacol 386: 1103-1110, 2013. 